\title{
Reliability and Accuracy of Menton Point in Determination of Facial Asymmetry and Mandibular Laterality
}

\author{
Rajkumar Maurya ${ }^{1 *}$, Harsh Ashok Mishra ${ }^{2}$ and Harpreet Singh ${ }^{3}$ \\ ${ }^{1}$ Department of Orthodontics,Army Dental Centre (R\&R), India \\ ${ }^{2}$ Department of Orthodontics, RishirajCollege of Dental Sciences and Research Cente, India \\ ${ }^{3}$ Department of Orthodontics, ESI Dental Hospital, India \\ *Corresponding author: Rajkumar Maurya, Department of Orthodontics,Army Dental Centre (R\&R), New Delhi,India
}

Submission: 㭗 May 18, 2018 ; Published: 侮 July 02, 2018

\begin{abstract}
Objectives:Assesment of reliability of the facial laterality and mandibular deviation in orthodontic patients with photograph and postero-anterior cephalogram using menton point.

Methods:Photographs and posteroanterior cephalogram of 120 subjects ( 35 males, 85 females) with class I, class II and class III malocclusion, between age group of 12 to 25 years were obtained. The frontal photo and posteroanterior cephalogram were analyzed for facial laterality and mandibular deviation. The obtained values were statistically analyzed to assess the facial asymmetry in orthodontic patients.

Results:class I \& II patient's menton deviation to left side were significant \& highly significant respectively statistically with no significance in class III. Male subjects showed $46 \%$ deviation of menton to left and $9 \%$ to right, it was statistically significant. In class I, 51\% showed left sided deviation of the soft tissue menton was significantly greater than the subjects with right sided deviation i.e. $21 \%$. chin deviation in class II subjects showed $44 \%$ with left side were not significant statistically. In class III, $54 \%$ showed laterality of the chin to left side, $19 \%$ right side and no chin deviation in $27 \%$, which is statistically significant $(\mathrm{p}<0.05)$.
\end{abstract}

Conclusion:Menton deviation with left sided laterality were seen in class I and class II but, in class III menton deviation to right side were observed. Soft tissue overlying the hard tissue tries to minimize the skeletal asymmetry. Soft tissue chin was deviated to left in most subjects. Gender difference in both skeletal and soft tissue asymmetry was not significant.

Keywords:Facial asymmetry; Menton; Laterality; Posteroanterior cephalogram

\section{Introduction}

Laterality of the craniofacial form is the degree of imbalance or deviation in both qualitative and quantitative features in structure or relationship and both causing facial asymmetry [1]. Clinical facial asymmetry in the craniofacial complex ranges from the barely detectable to gross discrepancies between the right and left half of the face [2]. Asymmetry in craniofacial areas can be recognized as differences in the size or relationship of the two sides of the face. This may be the result of discrepancies either in the form of individual bones or a malposition of one or more bones in the craniofacial complex. Mild asymmetries are invariably present even in the most pleasing faces [3]. It becomes necessary to discover such mild asymmetry so that the range of normal variation of asymmetry can be established. There are various reasons, which have been suggested as the possible causative factor. These include genetic, non-genetic as well as environmental influences.
The asymmetry may also be limited to the overlying soft tissues. In diagnosis of facial and dental asymmetries, a thorough clinical examination and radiographic examination are necessary to determine the extent of the soft tissue, skeletal, dental and functional asymmetry [4]. From the orthodontic aspects, asymmetries that are of special interestare those which might involve the occlusion. Facial asymmetry may be associated with a mandibular displacement and abnormal path of closure of the mandible is usually due to occlusal prematurity. Diagnosis of asymmetries in orthodontics is important and must be differentially diagnosed as being either the result of a skeletal asymmetry, asymmetry within the dental arches, discrepancies between centric occlusion and centric relation, or a combination. A detailed study of the various diagnostic records obtained on the patient is necessary in order to determine the cause, location, and extent of the asymmetry. This will enable the clinician to formulate the proper treatment plan. Photographic 
evaluation and use of the postero-anterior cephalogram is most reliable and important diagnostic tool. The photographic method is a quantitative method that is not only valid and reproducible but also non-invasive, convenient to use, low cost, less technique sensitive and taking soft tissue morphology in consideration $[5,6]$. There has been a scarcity of literature regarding documentation of facial laterality leading to asymmetry of face, a subject that also remains controversial. Hence, the purpose of this study was to assess the facial laterality and mandibular deviation in Angles Class I, Class II \& Class III orthodontic patients using menton point.

\section{Materials and Methods}

A sample of 120 patients (35 male and 85 female) was randomly selected from the OPD of the Department of Orthodontics and Dentofacial Orthopedics in Service Hospital. All patients were subjected to photographic and posteroanterior cephalogram evaluation before the beginning of treatment (Table 1). The subject selected had Angle's class I, class II or class III molar relation, who required orthodontic treatment, had full complement of teeth, no history of orthodontic treatment and no gross deformity of facial skeleton. Patients with marked craniofacial anomalies or syndrome, any systemic illness, pathology, undergone surgical orthodontic treatment were excluded.

Table 1: Subjects distribution according to malocclusion, sex and age.

\begin{tabular}{|c|c|c|c|c|c|}
\hline Group & No & Mean Age & SD & Males & Females \\
\hline Class I & 53 & 17.4 & 3.5 & 10 & 43 \\
\hline Class II & 41 & 17.6 & 7.6 & 15 & 26 \\
\hline Class III & 26 & 19.5 & 3.5 & 10 & 16 \\
\hline Total & 120 & 17.5 & 5.2 & 35 & 85 \\
\hline
\end{tabular}

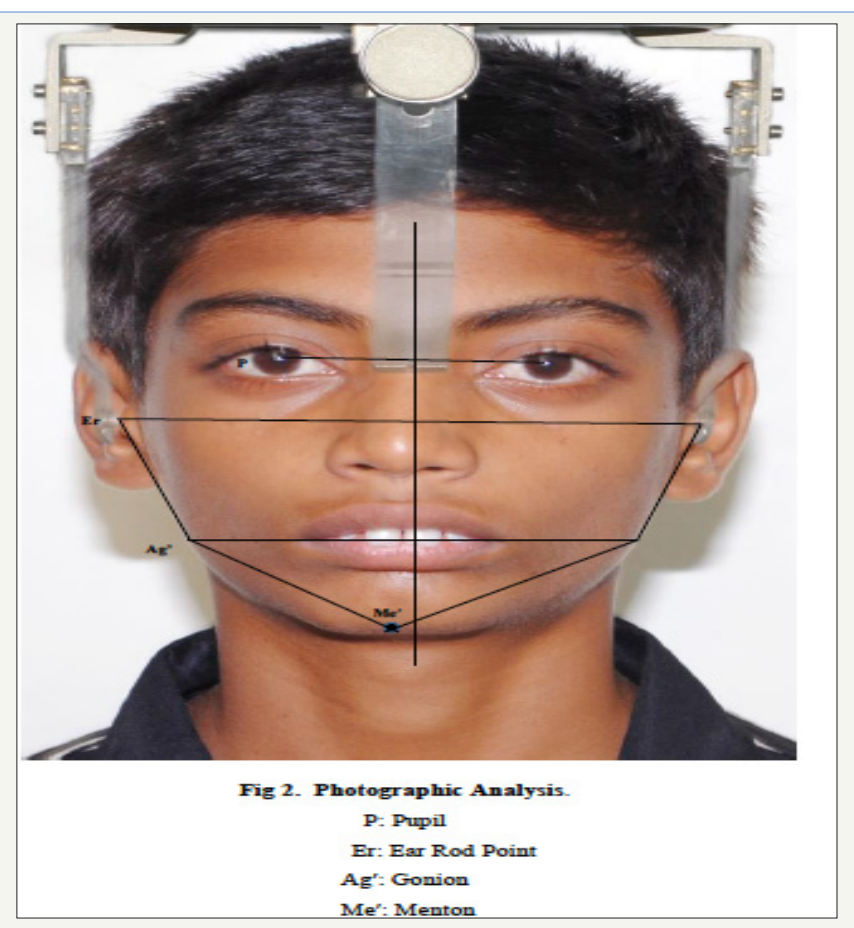

Figure 1: Photographic, Grummons Summary Frontal Analysis.

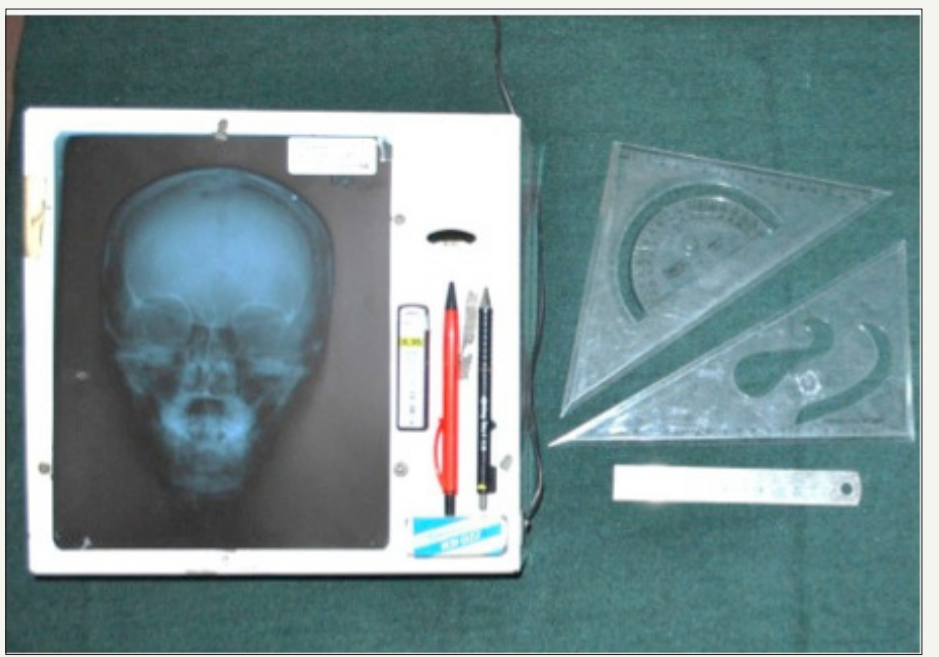

Figure 2: Armamentarium used in study. 
Facial photographs (Frontal view) were taken using Nikon D3000 SLR camera under standardized conditions with the head fixed using ear rods and the patient in natural head position (NHP). Facial photos analyzed for symmetry and laterality using photographic analysis (Figure 1) suggested by Haraguchi et al. [7] and Lee et al. [8]. Photos were taken by same photographer with constant shutter speed and aperture with focal length at $135 \mathrm{~mm}$ and distance from camera to patient of $160 \mathrm{~cm}$. Posteroanterior cephalogram of same 120 patients with natural head position (NHP) [9] were taken using standardized cephalometric technique taken with the same machine (GENDEX, ORTHORALIX 9200) in the PA projection with the distance of $2.0 \mathrm{~m}$ between the X-ray focus and the films [8]. The target to source distance of 5 feet and by the same radiographer with exposure factors $80 \mathrm{kvp}, 2.5$ seconds and $10 \mathrm{ma}$. They were developed for 30 seconds an fixed for 10 minutes and dried. The landmarks were identified, traced and analyzed (Figure 2). The cephalograms obtained were hand traced, various soft and hard tissue points identified and linear and angular measurements was carried out using Grummons summary frontal asymmetry analysis [10]; (Figure 1).

\section{Statistical Analysis}

All statistical analyses were carried out by using SPSS (Version 11.0, SPSS, Chicago, Ill). Analysis was done by using the arithmetic mean and the standard deviation for the mean was used as measure of deviation. Student's unpaired T-test was done to compare between males and females.

\section{Results}

Table 2 and Figure 3 shows mandibular offset at menton in Angles Class I, II \& III malocclusion. For class II patient's menton deviation to left side were highly significant statistically $(\mathrm{p}<0.001)$. The menton deviation in class I subjects to left were statistically significant $(\mathrm{p}<0.05)$. In class III patient's menton deviation were not significant statistically $(p>0.05)$. When males and females were compared for mandibular offset at menton, the male subjects showed $46 \%$ deviation of menton to left, $46 \%$ without menton deviation and only $9 \%$ showed menton deviation to right, it was statistically significant $(\mathrm{p}<0.05) .48 \%$ of the female subjects showed mandibular offset at menton to right side, which is highly significant statistically $(\mathrm{p}<0.001)$.

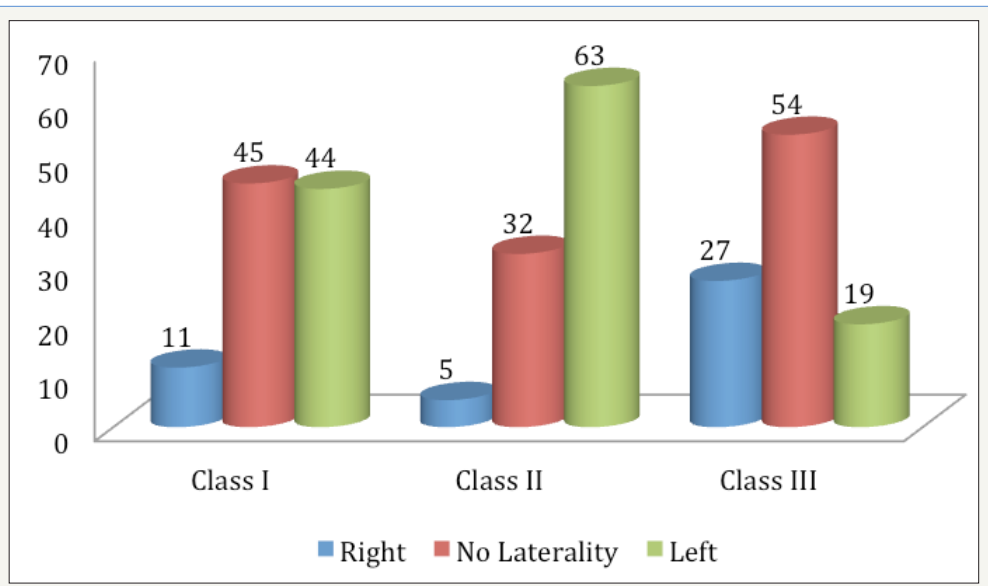

Figure 3: Subjects with mandibular offset at menton on left, right or no menton deviation from the facial midline subdivided by molar relation, Subjects with left, right or no chin deviation from the facial midline subdivided by molar relation.

Table 2: Subjects with mandibular offset at menton on left-or right-sided or no menton deviation from the facial midline subdivided by molar relation and sex.

\begin{tabular}{|c|c|c|c|c|c|}
\hline Group & Right & No Laterality & Left & $\mathbf{X}^{2}$ & P Value, sig \\
\hline Class I & $6(11)$ & $24(45)$ & $23(44)$ & 9.9 & 0.002 S \\
\hline Class II & $2(5)$ & $13(32)$ & $26(63)$ & 20.5 & $<0.001 \mathrm{HS}$ \\
\hline Class III & $7(27)$ & $14(54)$ & $5(19)$ & 1.3 & $0.24 \mathrm{NS}$ \\
\hline \multicolumn{7}{|c|}{ Gender Comparison } \\
\hline \multicolumn{7}{|c|}{ Males(n=35) } & $3(9)$ & $16(46)$ & $16(46)$ & 8.8 & $0.003 \mathrm{~S}$ \\
\hline Females(n=85) & $41(48)$ & $35(41)$ & $9(11)$ & 20.4 & $<0.001 \mathrm{HS}$ \\
\hline
\end{tabular}

Table 3 and Figure 4 shows the subjects with left or right or no chin deviation from the facial midline subdivided by molar relation and sex. In class I subjects, 51\% showed left sided deviation of the soft tissue menton was significantly greater than the subjects with right sided deviation which was $21 \%(\mathrm{p}<0.05)$. Whereas, the chin deviation in class II subjects showed $44 \%$ with left side deviation were not significant statistically ( $\mathrm{p}=0.05$ ). In class III, $54 \%$ of the subjects showed laterality of the chin to left side, $19 \%$ showed chin to right side and no chin deviation in $27 \%$, which is statistically significant $(\mathrm{p}<0.05)$. Male and female comparison of chin deviation 
showed $40 \%$ of chin laterality to left, $26 \%$ to right and no chin deviation in $34 \%$ in male subjects and was found to be statistically significant whereas in female subjects 53\% showed chin laterality to left, $18 \%$ to right and $29 \%$ without chin deviation which was highly significant statistically $(\mathrm{p}<0.001)$.

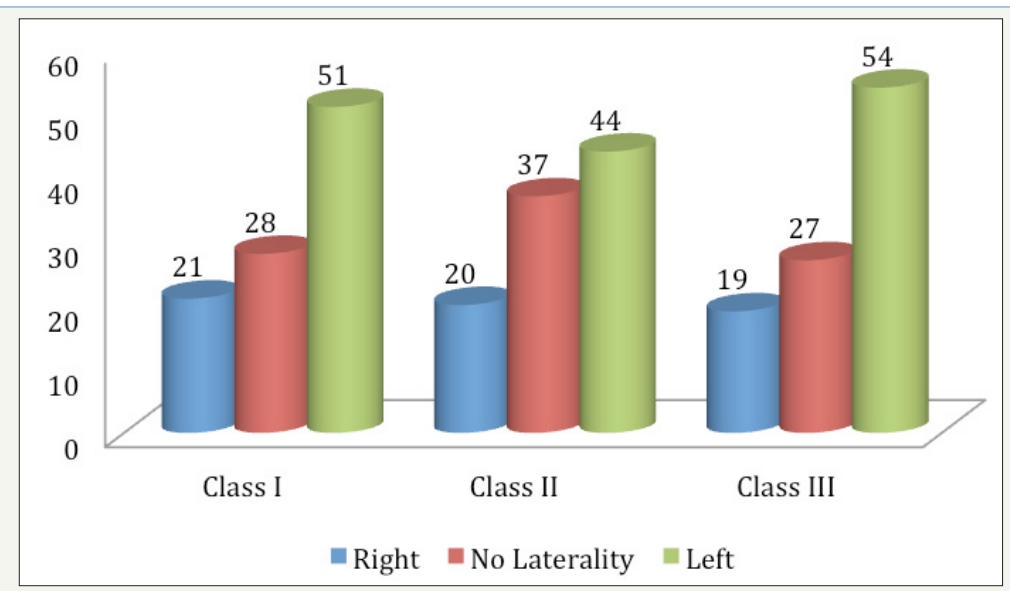

Figure 4: Subjects with mandibular offset at menton on left, right or no menton deviation from the facial midline subdivided by sex, Subjects with left, right or no chin deviation from the facial midline subdivided by sex.

Table 3: Subjects with left-or right-sided or no chin deviation from the facial midline subdivided by molar relation and sex.

\begin{tabular}{|c|c|c|c|c|c|}
\hline Group & Right & No Laterality & Left & X $^{2}$ & P Value, sig \\
\hline Class I & $11(21)$ & $15(28)$ & $27(51)$ & 6.7 & $0.009 \mathrm{~S}$ \\
\hline Class II & $8(20)$ & $15(37)$ & $18(44)$ & 3.8 & 0.05 NS \\
\hline Class III & $5(19)$ & $7(27)$ & $14(54)$ & 4.2 & $0.04 S$ \\
\hline \multicolumn{7}{|c|}{ Gender Comparison } \\
\hline Males(n=35) & $9(26)$ & $12(34)$ & $14(40)$ & 1 & 0.29 NS \\
\hline Females(n=85) & $15(18)$ & $25(29)$ & $45(53)$ & 15 & $<0.001$ HS \\
\hline
\end{tabular}

\section{Discussion}

The present study was conducted to evaluate and asses mandibular deviation and laterality of chin in 120 orthodontic patients (35 males and 85 females) divided into class I, class II and class III according to Angles molar relation, using standardized facial photographs and posteroanterior cephalometric measurements using Grummons [10] analysis. The main objective of the present study was to analyze the nature of deviation, in face and jaws with an effort to find the source of the problem. Mandibular deviation in the present study was represented by mandibular offset at menton. Menton deviated to left side in $44 \%$ in class I subjects, $63 \%$ in class II subject and $19 \%$ in class III subject. No laterality was seen in $45 \%, 13 \%$ and $54 \%$ in class I, class II and class III subjects respectively, whereas menton deviation to right side were seen in $11 \%$ in class I, $5 \%$ in class II, and $27 \%$ in class III. For class II patients menton deviation to left side were highly significant statistically $(p<0.001)$. The menton deviation in class I subjects to left were statistically significant $(\mathrm{p}<0.05)$. In class III patient's menton deviation were not significant statistically $(p>0.05)$. When males and females were compared for mandibular offset at menton, the male subjects showed $46 \%$ deviation of menton to left, $46 \%$ without menton deviation and only $9 \%$ showed menton deviation to right, it was statistically significant $(\mathrm{p}<0.05) .48 \%$ of the female subjects showed mandibular offset at menton to right side which is highly significant statistically $(\mathrm{p}<0.001)$. No deviation of menton was seen in $41 \%$ of the female patients whereas $19 \%$ showed left side deviation. The mean deviation of menton was found to be between $0.27 \mathrm{~mm}$ to $1.88 \mathrm{~mm}$ in class I, class II and class III group. The difference was statistically significant $(\mathrm{p}<0.05)$ between class I and class II group and class II and class III group, but the difference between class I and class III was not statistically significant. In the present study $57.5 \%$ chin deviation was noted, which is slightly lower than Severt and Proffit [11]. who found an incidence of $74 \%$ of chin deviation. The high incidence of chin deviation may be due to the asymmetries of mandibular length and gonial angle, which also showed high incidence.

In the present study, subjects with left-or right or no soft tissue chin deviation from the facial midline subdivided by molar relation and sex was studied. In class I subjects, 51\% showed left sided deviation of the soft tissue menton was significantly greater than the subjects with right sided deviation which was $21 \%(\mathrm{p}<0.05)$ and no deviation of chin in $28 \%$. Whereas, the chin deviation in class II subjects showed $44 \%$ with left side deviation were not significant statistically $(p=0.05)$. In class III, $54 \%$ of the subjects showed laterality of the chin to left side, $19 \%$ showed chin to right side and no chin deviation in $27 \%$, which is statistically significant $(\mathrm{p}<0.05)$. Male and female comparison of chin deviation showed $40 \%$ of chin laterality to left, $26 \%$ to right and no chin deviation in $34 \%$ in male 
subjects and was found to be statistically significant whereas in female subjects $53 \%$ showed chin laterality to left, $18 \%$ to right and $29 \%$ without chin deviation which was highly significant statistically $(\mathrm{p}<0.001) .69 .1 \%$ of the chin showed laterality to left or right side out of which $49 \%$ showed left side laterality. In a study of subjects with facial asymmetry, Haraguchi et al. [7] demonstrated $79.3 \%$ with chin deviation that had left sided laterality. While the proportion of subjects who exhibited no deviation at the menton decreased with age, the proportion of those having the left-sided deviation increased accordingly. In terms of skeletal pattern, no deviation at the menton was more frequently seen in subjects with the skeletal Class III malocclusion than in those with skeletal Class II malocclusion. Specifically, the proportions of the no-deviation, right-sided and left-sided groups in the skeletal Class II subjects were consistent for all pubertal growth periods. This is not in agreement with the present study.

The present study showed soft tissue menton deviation to left side in class I and class III subjects that were significant statistically $(p<0.05)$, class II subjects did not show any statistically significant result ( $p>0.05)$. This is in contrast to the study by Haraguchi et al. [7]. These findings suggest that overall; the proportion of subjects with jaw deviation at the menton remains unchanged during the pubertal growth period because those with skeletal Class II jaw relationship are likely to show relatively less growth of the mandible, even during the pubertal growth period. In contrast, skeletal Class III patients generally exhibit greater growth and also may be more likely to be affected by postnatal, environmental influences because of the relatively longer jaw growth period. Previous studies $[1,6,12]$ have discussed possible causes of facial laterality. Most have concluded that environmental influences were the most likely cause. Habitual chewing on one side has been reported to lead to increased skeletal development on the ipsilateral side [1,13] others have also discussed the possibility that such laterality is simply a response of functional adaptation to asymmetrical masticatory activity. According to a recent report, lateral displacement of the cephalometric menton toward the left side of the face is found more frequently than right sided deviation [14].

The study also documented, however, that subjects who had received chin cup treatment or had exhibited TMJ symptoms and/or reported a history of maxillofacial injury showed a higher proportion of right-sided chin deviation at menton when compared with those who had not experienced those factors. It was therefore suggested that these postnatal factors are not the causes of directional uniqueness in menton deviation. Given the possibility that the right-side hemiface grows wider than its counterpart because of postnatal factors, such as more use of a habitually preferred chewing side, it would be reasonable to assume that the proportion of individuals who show facial laterality toward the right-side increases during the pubertal growth period $[15,16]$.

In the present study, it has been observed that the malocclusions present with different type of significant asymmetries, which should be addressed when diagnosis and treatment planning is done. Our study clearly indicated that asymmetries are much higher in subjects with malocclusion, which demands the need for standardizing the method for quantification and classification of facial asymmetries.

\section{Conclusion}

Menton deviation with left sided laterality were seen in most of class II and class I cases but, in class III subjects menton deviation to right side were observed. Soft tissue overlying the hard tissue tries to minimize the skeletal asymmetry. Soft tissue chin was deviated to left in class I and class III subjects with lesser deviation in class II subjects. Gender difference in both skeletal and soft tissue asymmetry was not statistically significant.

\section{References}

1. Shah SM, Joshi MR (1978) An assessment of asymmetry in normal chronic facial complex. Angle Orthod 48(2): 141-148.

2. (1982) Stedman's Medical Dictionary ( $24^{\text {th }}$ edn) The Williams and Wilkins Company, Baltimore, USA, p. 134

3. Peck S, Peck L, Kataja M (1991) Skeletal asymmetry in esthetically pleasing faces. Angle Orthod 64: 43-48.

4. Pirttiniemi PM (1994) Association of mandibular and facial asymmetry: A Review. Am J Orthod Dentofac Orthop 106(2): 191-200.

5. Melnik AK (1992) A cephalometric study of mandibular asymmetry in a longitudinally followed sample of growing children. Am J Orthtod and Dentofac Orthop 101(4): 355-366.

6. Mulick JF (1965) An investigation of craniofacial asymmetry using serial twin study method. Am J Orthod 51(2): 112-129.

7. Haraguchi S, Iguchi Y, Takada K (2008) Asymmetry of face in orthodontic patients. Angle Orthod 78(3): 421-426.

8. Lee MS, Chung DH, Lee JW, Cha KS (2010) Assessing soft-tissue characteristics of facial asymmetry with photographs. Am J Orthod Dentofacial Orthop 138(1): 23-31.

9. Showfety KJ, Vij PS, Matteson S (1983) A simple method for taking natural head position cephalograms. Am J Orthod 83(6): 495-500.

10. Grummans DC, Copello KVD (1987) A frontal asymmetry analysis. J Clin Orthod 21(7): 448-465

11. Severt TR, Proffit WR (1997) The prevalence of facial asymmetry in the Dentofacial deformities in the population at the University of North Carolina. Int J Adult Orthod Orthognath Surg 12(3): 171-176.

12. Burke PH (1992) Serial observation of asymmetry in the growing face. Orthod 19(4): 273-285.

13. (2007) Proffit WR, White RR Sarver DM (Eds.), Contemporary treatment of dentofacial deformity, Mosby, Missouri, USA, p. 195.

14. Azevedo AR, Janson G, Fernando F, Henriques C, Freitas MR (2006) Evaluation of asymmetries between subjects with Class II subdivision and apparent facial asymmetry and those with normal occlusion. Am J Orthod Dentofacial Orthop 129(3): 376-383

15. Farkas LG, Cheung G (1981) Facial asymmetry in Healthy North American Caucasians. Angle Orthod 51(1): 76-78.

16. Schmid W, Mongini F, Felisio A (1991) A computer based assessment of structural and displacement asymmetries of the mandible. Am J Orthod Dentofac Orthop 100(1): 19-34 
Creative Commons Attribution 4.0 International License

For possible submissions Click Here

Submit Article
MRD Research
in Dentistry

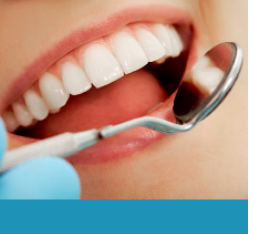

\section{Modern Research in Dentistry}

\section{Benefits of Publishing with us}

- High-level peer review and editorial services

- Freely accessible online immediately upon publication

- Authors retain the copyright to their work

- Licensing it under a Creative Commons license

- Visibility through different online platforms 